\title{
Chronische Bauchschmerzen ernst nehmen
}

\begin{abstract}
W enn Kinder chronisch unter Bauchschmerzen leiden, stimmt etwas nicht, auch wenn organisch alles in Ordnung ist", machte Dr. Martin Steinberger aus München deutlich.
\end{abstract}

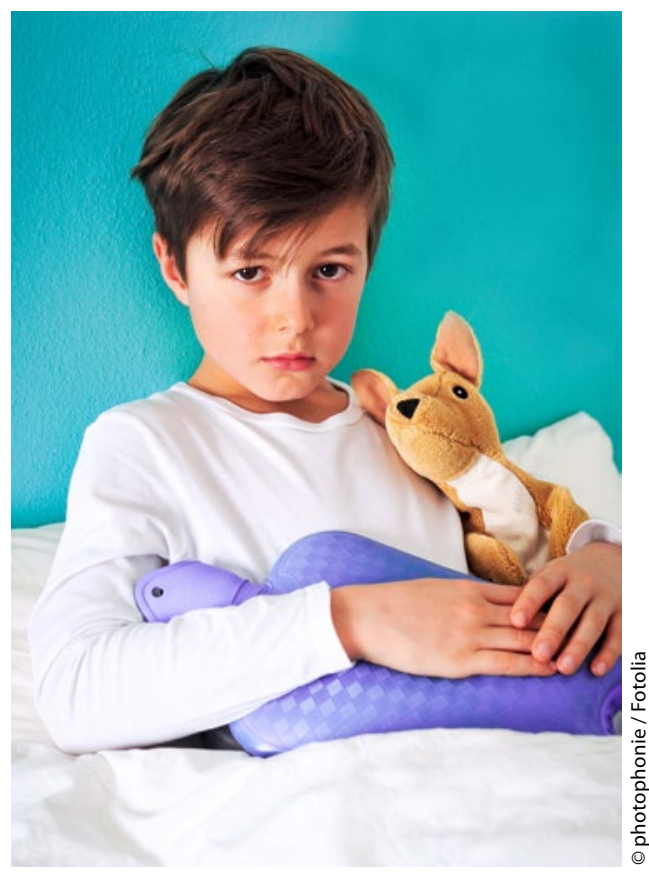

Häufig handelt es sich um eine dynamische Entwicklung von Faktoren, die sich gegenseitig beeinflussen und die Schmerzsymptomatik vorantreiben. So kann Bauchschmerz dazu führen, dass das Kind nicht lernen kann. Die Noten werden schlechter. Das gibt Ärger zu Hause, der wiederum die Schmerzen verstärkt. Auch Angst ist ein „Teufelskreisinduktor" und kann zur Chronifizierung von viszeralen Bauchschmerzen beitragen. Bei Kindern nicht zu unterschätzen ist der Einfluss der Familie, insbesondere der Mutter, auf das Schmerzverhalten. Wichtig ist eine adäquate Therapie, besonders weil Kinder mit chronischen Schmerzen ein hohes Risiko haben, auch als Erwachsene zu Schmerzpatienten zu werden.

Notwendig sei laut Steinberger eine interdisziplinäre, multimodale Schmerztherapie. Sie erfordert ein hohes Maß an Kooperation zwischen den einzelnen Therapeuten, nämlich ein gleichzeitiges inhaltlich, zeitlich und in der Vorgehensweise aufeinander abgestimmtes Vorgehen. Psychologen, Ärzte, Physiound Bewegungstherapeuten, optional auch Atem- oder Kunsttherapeuten sollen nach einem vorgegebenen Behandlungsplan eingebunden sein und ein gemeinsam abgestimmtes Therapieziel verfolgen. „Obligat ist eine gemeinsame Beurteilung des Behandlungsverlaufs innerhalb regelmäßiger vorgeplanter Teambesprechungen unter Einbindung aller Therapeuten."

Steinberger bemängelte, dass es für diese Therapieform noch wenig adäquate Angebote gibt, obwohl die bisher zur Verfügung stehenden Möglichkeiten vielversprechend sind. Seine Forderung: mehr spezialisierte Therapieangebote in allen Sektoren und im ganzen Bundesgebiet sowie eine adäquate wissenschaftlicher Auswertung der jeweiligen Konzepte. Als eine ambulante Therapieoption nannte er „Happy Pingu“, ein sechsstündiges verhaltenstherapeutisches Trainingsprogramm für Kinder im Grundschulalter mit begleitender Elternschulung.

Dr. Beate Fessler

Steinberger M. Multimodale Schmerztherapie bei Kindern mit chronischen Bauchschmerzen

\section{Niedriger Sozialstatus erhöht Risiko für psychische Störungen}

$\mathrm{D}$ ie im Jahr 2003 ins Leben gerufene Langzeitstudie KIGGS des RobertKoch-Instituts (RKI) beobachtet die seelische Entwicklung von Kindern und Jugendlichen in Deutschland. PD Dr. Thomas Lampert, Soziologe am RKI, beleuchtete den Zusammenhang zwischen Sozialstatus und Gesundheit: In der ersten Studienphase (Basiserhebung), wurde eine bundesweite repräsentative Stichprobe von 17.641 Kindern und Jugendlichen zu Gesundheitszustand, -verhalten und -versorgung sowie familiären Lebensbedingungen befragt und medizinisch untersucht. In den Jahren 2009-2012 (Welle 1, n = 12.368) wurde ein Teil der Studienteilnehmer erneut interviewt, gleichzeitig wurden jüngere Jahrgänge einbezogen. Auf Basis von beruflicher Bildung, Stellung und Einkommenssituation der Eltern ließen sich drei Statusgruppen abgrenzen: niedrig (20\%), mittel (60\%) und hoch $(20 \%)$.

Wie die Studienergebnisse belegen, wächst die Mehrheit der Kinder und Jugendlichen in Deutschland gesund auf. Ein deutlicher sozialer Gradient zeigte sich jedoch bei psychischen Auffälligkeiten wie Aufmerksamkeitsdefizit-/Hyperaktivitätsstörung (ADHS), Essstörungen, emotionalen Problemen und Gewalterfahrungen. Soziale Unterschiede fanden sich auch in Bezug auf das Gesundheitsverhalten, Ernährungsgewohnheiten, Bewegung, Mediennut- zung und Nikotinkonsum. Eltern mit niedrigerem Sozialstatus gingen mit ihren Kindern außerdem seltener zu den Früherkennungsuntersuchungen.

Wie Lampert weiter ausführte, macht sich der Einfluss des sozialen Status bereits sehr früh bemerkbar: „Wir sehen bei fast allen perinatalen Einflussfaktoren soziale Unterschiede, zum Beispiel beim Stillen oder mütterlichem Rauchen in der Schwangerschaft." Statusspezifische Unterschiede stellen deshalb hohe Anforderungen an Prävention und Gesundheitsförderung. Interessanterweise ließen sich bei akuten und chronischen Erkrankungen nur geringe Abweichungen zwischen den einzelnen Statusgruppen feststellen.

Dr. Martina-Jasmin Utzt

Lampert T. Einfluss des sozialen Status auf die Gesundheit von Kindern und Jugendlichen 